$\checkmark$ the main motivation for chasing the constant is simply that not having a reliable value is irksome. "It's hard to live with discrepancy," says Schlamminger. It is possible that $G$ appears to be so capricious because our basic understand嵌 ing of gravity is flawed, he says, but that seems unlikely.

Quinn points out that NIST is hosting this week's meeting, and he hopes that the institute will provide initial funding for the project, but nothing is yet firm. Assuming that cash is found, the consortium could have a reliable value for $G$ in the next five years, says Quinn, or at least a good handle on where disagreements lie. "There must be a solution to this," he says, "there must be." -

\section{TROUBLE WITH BIG G}

In 2000, scientists measured the gravitational constant, $G$, with smaller error bars than ever before. But since then, a variety of experiments using different techniques have produced a range of values - and uncertainty in the official CODATA* value has increased since 2006 .

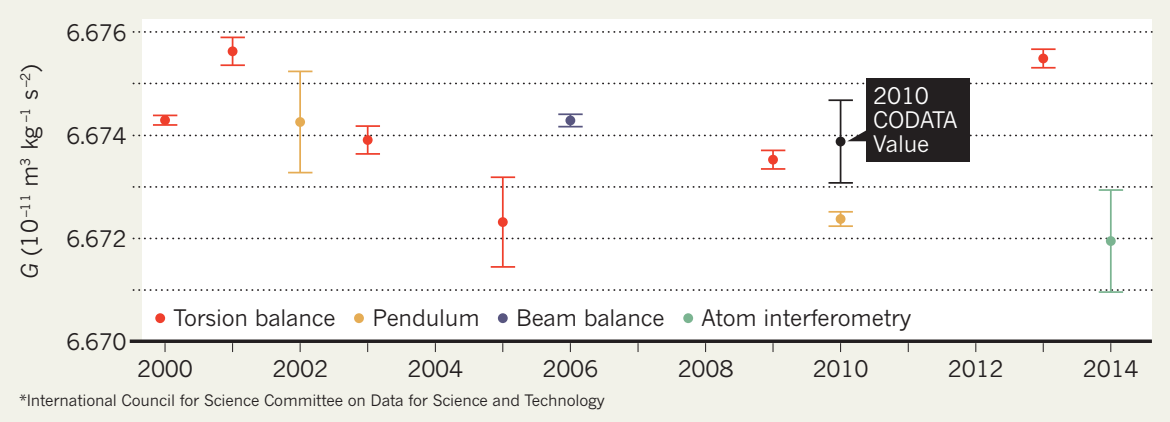

\title{
Marmosets are stars of Japan's ambitious brain project
}

Ten-year brain-mapping effort will use monkeys to study human neural and mental disorders.

\section{BY DAVID CYRANOSKI}

$\mathrm{E}$ urope has one, the United States has one. Now Japan has thrown its hat into the neuroscience ring with the launch of its own brain-mapping project.

Unlike its Western counterparts, Japan's effort will be based on a rare resource - a large population of marmosets that its scientists have developed over the past decade - and on new genetic techniques that might be used to modify these highly social animals. The goal of the ten-year Brain/MINDS (Brain
Mapping by Integrated Neurotechnologies for Disease Studies) project is to map the primate brain to accelerate understanding of human disorders such as Alzheimer's disease and schizophrenia. On 11 September, the Japanese science ministry announced the names of the group leaders - and how the project would be organized.

Funded at $¥ 3$ billion (US $\$ 27$ million) for the first year, probably rising to about $¥ 4$ billion for the second, Brain/MINDS is a fraction of the size of the European Union's Human Brain Project and the United States' BRAIN (Brain

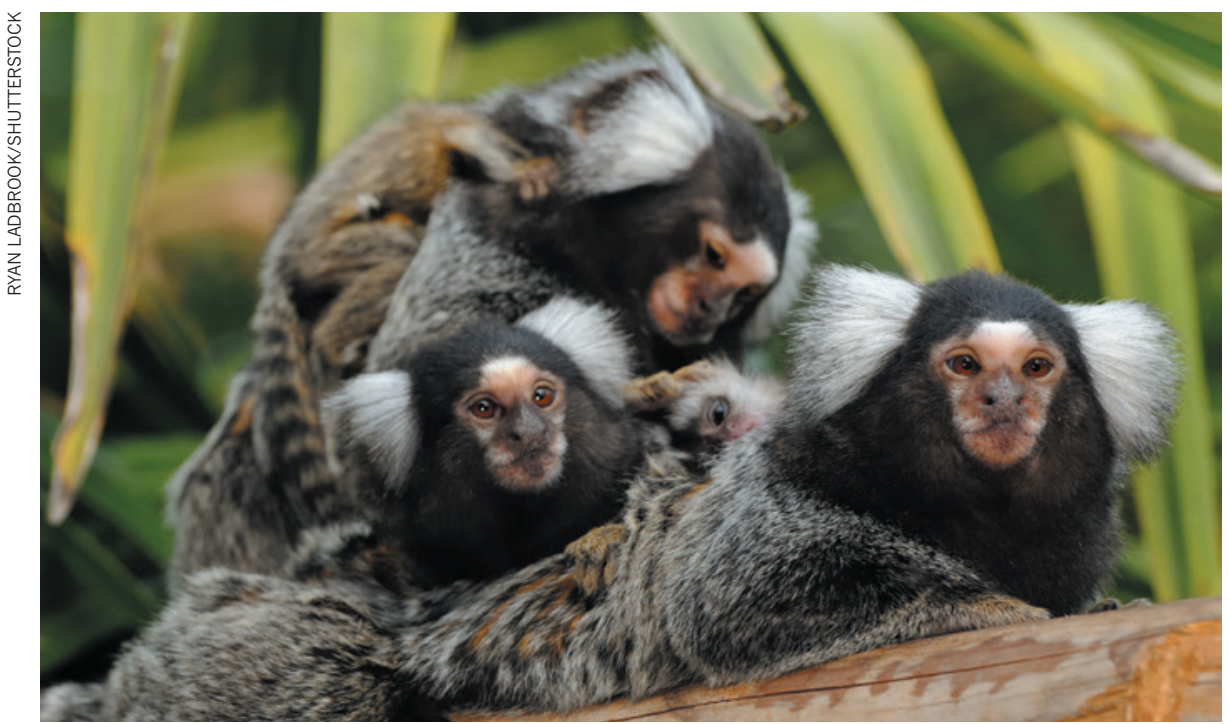

Marmosets share behaviours, such as making eye contact as a means of communication, with humans.
Research through Advancing Innovative Neurotechnologies) Initiative, both of which are projected to receive at least US $\$ 1$ billion over the next decade. But researchers involved in those efforts say that Brain/MINDS fills a crucial gap between disease models in smaller animals that too often fail to mimic human brain disorders, and models of the human brain that need validating data.

"It is essential that we have a genetic primate model to study cognition and cognitive brain disorders such as schizophrenia and depression, for which we do not have good mouse models," says neuroscientist Terry Sejnowski at the Salk Institute in La Jolla, California, who is a member of the National Institutes of Health BRAIN Initiative Working Group. "Other groups in the United States and China have started transgenic-primate projects, but none is as large or as well organized as the Japanese effort."

Neuroscientist Henry Markram at the Swiss Federal Institute of Technology in Lausanne, who heads the European initiative, also welcomes the effort: "It is absolutely impressive, and Japan should be applauded for putting together an incredible plan."

Central to the Brain/MINDS effort is the creation of transgenic marmosets to elucidate cognitive function and as models of human brain disease. Although more distantly related to humans than primates such as chimpanzees, these monkeys are in many ways ideal for brain research. Their small size and fecundity make them easier and more efficient to work with 
than, say, macaques, which are commonly used as animal models.

The marmoset brain is compact, too - just 8 grams, making it relatively easy to analyse. Yet the frontal lobe - a brain area involved in human psychiatric disease - is more developed in marmosets and more like the human version than the frontal lobes of other animals with similarly small brains. Marmosets also share behavioural characteristics with humans that other monkeys, and even chimps, do not, such as living in family units that resemble human ones and making eye contact as a means of communication, rather than as a form of aggression.

The monkeys are thus expected to be a good model for studying conditions such as Parkinson's disease and Alzheimer's disease. And learning what causes the breakdown of social behaviours such as making eye contact might help to clarify the mechanisms underlying autism, suggests Katsuki Nakamura, a neuroscientist at Kyoto University in Japan who works with marmosets.

Recent advances could also help researchers to genetically engineer marmosets more efficiently. In 2009, a team led by transgenic-animal specialist Erika Sasaki at the Central Institute for Experimental Animals in Kawasaki, Japan, was the first to introduce a gene into a primate that was passed on to viable offspring.

A gene-editing method known as CRISPR now permits precise DNA alterations ${ }^{1,2}$ and has been used to create transgenic monkeys ${ }^{3}$.

At the same time, geneticists have identified some of the key mutations that contribute to human disorders such as schizophrenia and autism. "This is a very important time to be developing primate genetic models," says Robert Desimone, director of the McGovern

"I would love to see one single important disease studied in great depth." Institute for Brain Research at the Massachusetts Institute of Technology in Cambridge.

Brain/MINDS will be divided into three groups. One, led by project co-leader Hideyuki Okano at Keio University, will map brain function to structure using techniques such as functional magnetic resonance imaging. The group will use transgenic models of disease to link studies of macro-scale functions - longdistance pathways across the brain - to microscale studies that identify and characterize specific neuronal features. The second group, led by the project's other co-leader, Atsushi Miyawaki at the RIKEN Brain Science Institute in Wako, and consisting of 17 independent teams, will develop technologies to support the mapping effort. And the University of Tokyo's
Kiyoto Kasai will lead the third group, which will collect information, such as brain scans, from human patients. This will can be used to find signatures of psychiatric, neurological and vascular disease to feed back to the marmoset researchers.

Japan's rules regarding the use of primates in such research are less stringent than European or US regulations, but the project could still face ethical hurdles over issues of cruelty to animals. "It will be important for the Japanese marmoset project to carefully examine the ethical issues that will inevitably arise up the road," says Sejnowski.

BRAIN/minds is technically ambitious. Neuroscientist Afonso Silva at the National Institute of Neurological Disorders and Stroke in Bethesda, Maryland, who collaborates with Sasaki, points out that CRISPR has yet to be shown to work in marmosets. He adds that the project's researchers must resist the temptation to tackle too many conditions, so as not to spread themselves too thinly. "I would love to see one single important disease studied in great depth," he says. "Validating the approach on that one disease will then enable the project to be repeated in other diseases." -

1. Cong, L. et al. Science 339, 819-823 (2013)

2. Mali, P. et al. Science 339, 823-826 (2013).

3. Niu, Y. et al. Cell 156, 836-843 (2014).

\section{Blue LED wins physics Nobel}

\section{Invention revolutionized lighting and will reduce global electricity consumption.}

\section{BY ELIZABETH GIBNEY}

$\mathrm{F}$ ound in smartphones, computer screens and energy-efficient bulbs, blue lightemitting diodes (LEDs) are everywhere. That they have now earned three Japaneseborn inventors the 2014 Nobel Prize in Physics is a rare example of the award being given for a practical invention.

LEDs are devices that emit light when subjected to an electric current. Green and red versions have been around since the 1950s, but creating blue-emitters was a technical challenge that stumped industry efforts for decades.

It was not until the 1990s that engineers Isamu Akasaki and Hiroshi Amano, both at Japan's Nagoya University, in parallel with electrical engineer Shuji Nakamura, then working at Japanese chemicals firm Nichia, succeeded in creating a blue LED.

LEDs are sandwiches of semiconductor materials. The layers are 'doped' with other elements, which provides some layers with extra electrons and others with a surplus of 'holes',
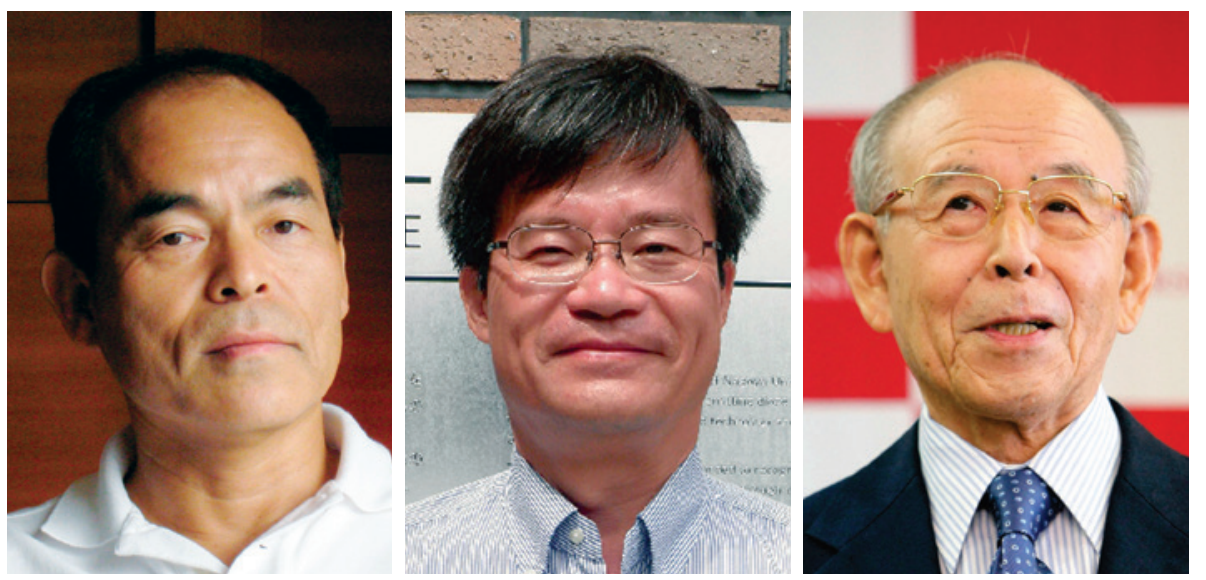

Shuji Nakamura, Hiroshi Amano and Isamu Akasaki (left to right) won the 2014 Nobel Prize in Physics.

where missing electrons leave behind a positive charge. When an electrical current is applied, the electrons and holes combine at the junctions between the layers and emit light as a result.

From the 1980s, physicists focused their efforts on the material gallium nitride as a target for making high-power blue LEDs, but they were faced with several technological hurdles. One was creating thin, high-quality crystals of the material, which are notoriously difficult to grow. Another was doping gallium nitride such that it emitted light efficiently. 